\title{
Computational Studies of the Farnesyltransferase Ternary Complex Part II: The Conformational Activation of Farnesyldiphosphate ${ }^{\dagger}$
}

\author{
Guanglei Cui and Kenneth M. Merz Jr. \\ Department of Chemistry and the Quantum Theory Project, 2328 New Physics Building, PO Box \\ 118435, University of Florida, Gainesville, FL 32611-8435
}

\begin{abstract}
Studies aimed at elucidating the reaction mechanism of farnesyltransferase (FTase), which catalyzes the prenylation of many cellular signaling proteins including Ras, has been an active area of research. Much is known regarding substrate binding and the impact of various catalytic site residues on catalysis. However, the molecular-level details regarding the conformational rearrangement of farnesyldiphosphate (FPP), which has been proposed via structural analysis and mutagenesis studies to occur prior to the chemical step, is still poorly understood. Following on our previous computational characterization of the resting state of the FTase ternary complex, the thermodynamics of the conformational rearrangement step in the absence of magnesium was investigated for the wild type FTase and the Y300F $\beta$ mutant complexed with the peptide CVIM. In addition, we also explored the target dependence of the conformational activation step by perturbing isoleucine into a leucine (CVLM). The calculated free energy profiles of the proposed conformational transition confirm the presence of a stable intermediate state, which was identified only when the diphosphate is monoprotonated $\left(\mathrm{FPP}^{2-}\right)$. The farnesyl group in the computed intermediate state assumes a conformation similar to that of the product complex, particularly for the first two isoprene units. We found that $Y 300 \beta$ can readily form hydrogen bonds with either of the phosphates of FPP. Removing the hydroxyl group on $\mathrm{Y} 300 \beta$ does not significantly alter the thermodynamics of the conformational transition, but shifts the location of the intermediate farther away from the nucleophile by $0.5 \AA$, which suggests that $Y 300 \beta$ facilitate the reaction by stabilizing the chemical step. Our results also showed an increased transition barrier height for CVLM (1.5 kcal/mol higher than that of CVIM). Although qualitatively consistent with the findings from the recent kinetic isotope experiments by Fierke and coworkers, the magnitude is not large enough to affect the rate limiting step.
\end{abstract}

\section{Keywords}

Farnesyltransferase; reaction mechanism; free energy profile; conformational rearrangement

\section{INTRODUCTION}

Farnesyltransferase (FTase) is a key enzyme in the cellular RTK (receptor tyrosine kinase) signal transduction pathway. It recognizes the $\mathrm{CaaX}(\mathrm{a}=$ aliphatic, $\mathrm{X}=\mathrm{Met})$ motif found at the C-terminus of protein acceptors (Ras, Rab, Rho, etc.) and chemically modifies them with farnesyl, a 15 carbon atom long prenyl group (Figure 1), which significantly enhances the

\footnotetext{
${ }^{\dagger}$ This work was supported by NIH grant GM44974 to K. M. M., Jr.

*To whom correspondence should be addressed: Department of Chemistry and the Quantum Theory Project, 2388 New Physics Building, PO Box 118435, University of Florida, Gainesville, FL 32611-8435. Tel: (352) 392-6973. Fax: (352) 392-8722. Email: merz@ qtp.ufl.edu.
} 
affinity of these proteins for membrane interfaces where extracellular signals are accepted and processed (1-6). In light of the critical position of FTase in the RTK signal transduction pathway and the connection between malfunctioning Ras proteins upon mutation and tumor formation, FTase has emerged as a major anti-cancer drug target with several drug candidates being evaluated in clinical trials (7-12).

The mechanism of FTase-catalyzed farnesylation has been the subject of extensive biochemical and biophysical research since the discovery of its important biological function. Steady-state kinetics $(13,14)$, transient kinetics (15), site-directed mutagenesis (14), and X-ray crystallography (16-22) all have made considerable contributions to the understanding of the mechanism. From these studies, FTase was identified as a heterodimeric zinc-metalloenzyme, first forming a binary complex with FPP $\left(\mathrm{K}_{\mathrm{d}} \sim 5 \mathrm{nM}\right.$, PDB: 1FT2), which subsequently accommodates the protein substrate. Both farnesyl and the farnesyl acceptor (a CaaX box at the $\mathrm{C}$-terminus of the protein substrate, $\mathrm{a}=$ aliphatic, $\mathrm{X}=\mathrm{Met}$ ) are located at the solventaccessible interface between the $\alpha$ and $\beta$ subunits. The ternary complex structure (PDB ID: 1QBQ) of FTase and $\alpha$-hydroxyfarnesylphosphonic acid (HFP) and a tetrapeptide (Ace ${ }^{1}$ $\mathrm{Cys}^{2}-\mathrm{Val}^{3}-\mathrm{Ile}^{4}-\mathrm{Mse}^{5}$ ) reveals that the farnesyl moiety and the tetrapeptide are bound in the same binding site pocket surrounded with hydrophobic residues while the diphosphate group of FPP is positioned next to a highly positively charged pocket, comprising of $\mathrm{K} 164 \alpha$, R291 $\beta$, and K294 $\beta$. Mutation studies suggest these basic residues as well as H248 $\beta$ and Y300 $\beta$ are important for FPP binding (Figure 2). Strikingly, the nucleophile $\mathrm{Cys}^{2}$ of the tetrapeptide bound to the divalent zinc ion, is $7.2 \AA$ away from the C1 carbon of FPP suggesting the presence of intermediate state along the reaction pathway that features a conformationally activated cofactor. The recently solved structure of the farnesylated product complex (PDB ID: 1KZP) did not indicate any major conformational changes of FTase ternary complex, and thereby, inspired the hypothesis that rotation of the bond between the first two isoprenoid groups reduced the distance between the electrophile and $\mathrm{Cys}^{2}$, which we will term the "FPPapproaching mechanism". However, as an important step in the reaction mechanism of FTase, the structure of this intermediate state (if ever present) has not been identified. Recently, both ${ }^{3} \mathrm{H}$ - and ${ }^{14} \mathrm{C}$ - kinetic isotope effects (KIE) in FTase were measured for the peptides TKCVIF and GCVLS (23). The $\alpha$-secondary ${ }^{3} \mathrm{H}$-KIE of TKCVIF in the absence of magnesium $(1.151 \pm 0.006)$ corroborated the concerted mechanism $\left(\mathrm{S}_{\mathrm{N}} 2\right.$-like) and the partially formed carbenium ion character at the transition state, even though the primary ${ }^{14} \mathrm{C}-\mathrm{KIE}$ was less indicative. On the other hand, the same KIE measurement for GCVLS obtained under the same conditions was only $1.00 \pm 0.04$, which indicates that the rate-limiting step in farnesylation was shifted, owing to the difference in target sequence, possibly from the chemical reaction step to the conformational activation step. The lack of any information regarding either the structure of this hypothetical intermediate state or the energetic cost of this conformational activation step compromises our interpretation of available experimental results. Despite the tremendous interest in the nature of this intermediate, no research has been reported, to the best of our knowledge, from both experimental and computational studies that provide molecular-level details regarding its structure. We note that at the time of the writing a second kinetic isotope experiment was reported by Distefano and co-workers (24) using ${ }^{2} \mathrm{H}$ and ${ }^{13} \mathrm{C}$ isotopes, which supports the same reaction mechanism. In addition, a model of the transition state (TS) of the chemical event was proposed based on KIE calculations on model compounds.

With experimental structural information regarding the intermediate state lacking, computer simulation techniques combined with an accurate molecular mechanics model will allow us to probe the presence of the intermediate state theoretically. The recent heavy-atom KIE measurements (23) are important additions to the current understanding of FTase catalysis because it not only provides the definitive proof for the proposed concerted reaction mechanism but also sheds light on the energetic penalty of the conformational activation step. In the second part of our computational studies on FTase catalyzed prenylation, we extended the molecular 
mechanics investigation reported earlier in Part I and focused on identifying the hypothetical intermediate state and computing the free energy change of the conformational transition in terms of the potential of mean force (PMF) (25) in the absence of magnesium, from which the system-dependent energetics of the activation revealed in the KIE experiments can be directly examined.

Previously, we carefully calibrated the force field parameters of the backbone rotations of the farnesyl group, with which we were able to compute the ensemble averaged J-coupling constant for one backbone dihedral angle in excellent agreement with experiment (26). Subsequently, a molecular dynamics (MD) simulation of the FTase ternary complex ( $\mathrm{FPP}^{3-}$ and the peptide CVIM) was carried out in explicit solvent for over 6 nanoseconds, during which only the resting state was sampled and the diphosphate was locked in its inactive form by the positively charged binding pocket consisting of R291 $\beta$, K294 $\beta$, and K164 $\alpha$. We found that the dynamics of $\mathrm{FPP}^{3-}$ binding and the structural details of the binding site were in general consistent with the findings from mutagenesis studies. However, the $\mathrm{pH}$-dependent kinetics experiments suggested that FPP may be mono-protonated in the ternary complex, the $\mathrm{pK}_{\mathrm{a}, 3}$ of the last proton of the diphosphate being 7.4 estimated from a 6-state kinetic model (27). Because diphosphate binding is likely to be dominated by electrostatic interactions, reducing the charge of the diphosphate from -3 to -2 may have a significant impact on the energetic cost of conformational activation and substrate binding, and therefore both scenarios have been investigated herein. Our calculations on the potentials of mean force were able to identify the missing intermediate state in the $\mathrm{FPP}^{2-}$-bound scenario, but found closing the $7 \AA$-gap between the reactants was too high for $\mathrm{FPP}^{3-}$. It suggests that in the absence of magnesium FTase is only productive with $\mathrm{FPP}^{2-}$ bound. The correct charge assignment enabled us to compare the activation free energy profiles of the two peptides studied in the KIE experiments. We found that changing $\mathrm{Ile}^{3}$ to Leu increased the activation barrier height by about $1.5 \mathrm{kcal} / \mathrm{mol}$, which is qualitatively consistent with the interpretation made by Fierke and coworkers based on the KIE measurements. Moreover, we examined the impact of a mutation $\mathrm{Y} 300 \beta \mathrm{F}$ on the activation energetics. Early mutagenesis and kinetic studies using farnesylmonophosphate (FMP) showed that $\mathrm{Y} 300 \beta$ adversely affects the reaction rate constant $\left(k_{\text {chem }}\right)$ by 500 fold under optimal catalytic conditions if changed to phenylalanine (15). Since the reaction rate constant measured experimentally includes the contribution from both the conformational activation and the nucleophilic attack, the role of $Y 300 \beta$ warrants further study in order to separate the conformational transition cost from the chemical step.

In the following sections, the details about the various calculations carried out herein are given followed by a discussion of the results. In the end, we will amalgamate the findings of extant mechanistic studies with ours from the potential of mean force calculations with the goal of obtaining an improved molecular-level view of the catalytic mechanism of FTase.

\section{METHODS}

The general system setup and the protocol for the MD simulation of the FTase ternary complex (FPP and CVIM) have been described elsewhere (26), and were used in this study with little modification. As before, all the molecular dynamics simulations and potential of mean force calculations were carried out using the sander module in AMBER8 (28). Quantum mechanical calculations required by the force field parameterization were conducted using Gaussian 03 (29). In this paper, we will focus on the simulation of the FTase ternary complex with FPP $^{2-}$, where some modifications were necessary, and the details of the potential of mean force calculations of four different systems, including FTase/FPP ${ }^{3-} / \mathrm{CVIM}$, FTase/FPP ${ }^{2-} / \mathrm{CVIM}$, FTase/FPP ${ }^{2-} / \mathrm{CVLM}$, and FTase-Y300/F//FPP ${ }^{2-} / \mathrm{CVIM}$. 


\section{The MD Simulation of the FTase Ternary Complex with FPP2-}

Methyldiphosphate $\left(\mathrm{MPP}^{2-}\right)$ was used to model the mono-protonated FPP during parameterization with the hydrogen atom located on the $\beta$-phosphate. If positioned on the $\alpha$ phosphate, the hydrogen atom would transfer to the $\beta$-phosphate during gas phase QM optimization using the B3LYP/6-31+G* basis set. The most stable gas phase conformation of the $\beta$-protonated diphosphate is strongly favored by an intramolecular hydrogen bond, the strength of which would be attenuated in aqueous solution or the interior of protein due to competing interactions with water molecules or diphosphate binding residues. Hence, multipleconformation RESP (30) charge fitting is necessary if under-representation of other possibilities is to be avoided. We systematically scanned the potential energy surface of $\mathrm{MPP}^{2-}$ described by the two torsion angles involving the bridging oxygen atom (60-degree interval for each torsion) while the other degrees of freedom were optimized using B3LYP/ $6-31+\mathrm{G}^{*}$. A total of 30 conformations (a limitation set by the RESP program) including all the major minima, equally weighted, were used in the following charge calculation. The results are available in the supplementary material. The Gaff generic AMBER force field (31) for organic compounds, with additional torsion corrections obtained previously (26), was employed for the bonding (bond, angle, and torsion) and van der Waals interactions.

However, we found that it was difficult to maintain a stable MD calculation of the $\beta$-protonated diphosphate, even though multiple-conformation fitted charges were used. We observed from our test runs of $\mathrm{FPP}^{2-}$ in the gas phase as well as in explicit solvent that the structure was severely distorted and the dynamics failed to continue as soon as the intramolecular hydrogen bond was broken. The source of this failure was identified to be excessive 1-4 electrostatic interactions between the $\beta$-proton $(+0.3899 e)$ and the bridging oxygen atom $(-0.5717 e)$. This is likely a manifestation of the inadequacy of the underlying fixed-charge model used in the AMBER force field. In order to avoid possible complications in our subsequent analyses, the problem was corrected by increasing the scaling constant for the 1-4 electrostatic interactions for the $\beta$-proton to 2.25 (we note that this value is closer in magnitude to the 2.0 value used in the original AMBER force field, hence, this modification is not unprecedented) as opposed to the default value of 1.2 used for the rest of the system. This was the smallest and simplest change that produced stable dynamics for the $\beta$-protonated diphosphate, which allowed us to generate an $8.4 \mathrm{~ns}$ MD trajectory of the FTase/FPP ${ }^{2-} / \mathrm{CVIM}$ ternary complex at $300 \mathrm{~K}$ following the same procedure used in our previous study.

\section{The Potential of Mean Force Calculations of Various FTase Ternary Complexes}

The potential of mean force as a function of the distance (D) between the center of mass of atoms $\mathrm{C} 1, \mathrm{C} 2$, and $\mathrm{O} 1$ of $\mathrm{FPP}^{3-}$ or $\mathrm{FPP}^{2-}$ and $\mathrm{S}_{\gamma}$ of $\mathrm{Cys}^{2}$ of the target peptides were calculated using the umbrella sampling technique and the weighted histogram analysis method (WHAM) (32). 15 MD simulations were conducted with harmonic biasing potentials, whose centers were placed evenly along the reaction coordinate over the range of 4.5 to $8 \AA$ with an interval of $0.25 \AA$. This is possibly the largest portion of the reaction coordinate that can be investigated using a classical molecular mechanic potential, as the sum of the van der Waals radii of carbon and sulphur atoms in AMBER $f f 94$ force field (33) is approximately $4 \AA$. Because only the structure of the resting state is available, we first created the starting structure for each sampled window of the reaction coordinate via a slow scanning MD run with a relatively large harmonic driving potential along the reaction coordinate. The temperature was maintained at $300 \mathrm{~K}$ via a Berendsen thermostat (34). After the starting structures along the reaction coordinate were obtained, Anderson temperature coupling (35) was switched on for umbrella sampling. The same biasing strength $\left[20.0 \mathrm{kcal} /\left(\mathrm{mol} \cdot \AA^{2}\right)\right]$ was used for all of the umbrella MD simulations, each of which was run for at least $6 \mathrm{~ns}$ with a timestep of $2 \mathrm{fs}$ and over 300,000 values at each point along the reaction coordinate were saved. Only the last 4ns were used in Grossfield's implementation (36) of the WHAM analysis in order to reconstruct the unbiased distribution 
of the reaction coordinate, from which the free energy profile of the conformational transition was extracted. The convergence criterion of the WHAM analysis was set to $0.001 \mathrm{kcal}(37)$. The statistical uncertainties of the calculated potential of mean force were estimated by a Monte Carlo bootstrap analysis. A few extra nanoseconds of biased MD simulations were carried out, if necessary, to increase the sampling statistics at selected distances, particularly near the intermediate state and the peak of the transition barrier. We note that extensive sampling was critical to the identification of the intermediate states.

The PMF calculations were first performed for FTase/FPP ${ }^{3-} / \mathrm{CVIM}$ and FTase/FPP ${ }^{2-} / \mathrm{CVIM}$. For the PMF calculations of FTase/FPP ${ }^{2-} / \mathrm{CVLM}$ and FTase-Y300ßF/FPP ${ }^{2-} / \mathrm{CVIM}$, the initial structures for starting the biased umbrella MD simu; ations were built with the LEaP module of AMBER8 using the snapshots taken from the umbrella MD trajectories of FTase/FPP ${ }^{2-}$ / CVIM as templates. After the corresponding residues were replaced, the structures were minimized to remove van der Waals clashes introduced by the mutations and then relaxed at $300 \mathrm{~K}$ for $2 \mathrm{~ns}$ with the harmonic restraints. The same steps were followed in the production runs as described above.

\section{RESULTS AND DISCUSSION \\ The MD Simulation of FTase/FPP2-/CVIM}

The dynamics of the FTase/FPP ${ }^{2-} /$ CVIM ternary complex was simulated for $8 \mathrm{~ns}$. The change in the overall FTase conformation caused by the difference in the FPP protonation state was minimal. The backbone root mean square deviation (rmsd) from the initial structure is $1.4 \pm 0.2 \AA$ for FTase/FPP ${ }^{2-} /$ CVIM over the last $6 \mathrm{~ns}$, similar to the $1.3 \pm 0.2 \AA$ reported earlier for the FTase/ $\mathrm{FPP}^{3-} / \mathrm{CVIM}$ ternary complex. The average backbone conformations extracted from the MD trajectories of FTase/FPP ${ }^{2-} /$ CVIM and FTase/FPP ${ }^{3-} / \mathrm{CVIM}$ differ from each other by $1.0 \AA$. However, reducing the negative charge on the diphosphate weakened the interactions of the diphosphate with protein binding groups, and as a result, different hydrogen bonding networks were observed. While the strong attraction between the opposite charges allowed the diphosphate to form hydrogen bonds with all three positive side chains, K164 $\alpha$, R291 $\beta$ and $\mathrm{K} 294 \beta$ in FTase/FPP ${ }^{3-} / \mathrm{CVIM}$, it was not sufficient in the FTase/FPP ${ }^{2-} / \mathrm{CVIM}$ complex to counteract the repulsive interactions generated by bringing together the positive residues. This led to the loss of K294 $\beta$ in the hydrogen bonding network, which was subsequently replaced with the neutral residue Y300 $\beta$. The hydrogen bonding with Y300 $\beta$ was not observed in the previous MD simulation of FTase/FPP ${ }^{3-} / \mathrm{CVIM}$, but has been seen in the crystal structure of the FPP-bound FTase binary complex (PDB ID: 1FT2). Fierke and coworkers proposed that Y $300 \beta$ mainly interacts with the $\alpha$-phosphate based on kinetic studies (15), in which FMP was used as substrate, rather than the $\beta$-phosphate as observed in 1FT2 (17). We found that the hydroxyl group of $Y 300 \beta$ is statistically closer to the $\beta$-phosphate during the last 6 ns of the MD simulation judged by the distances between the oxygen atom of the donor and the oxygen atoms of the diphosphate. However, the bridging oxygen atom certainly is within hydrogen bonding range with an average distance of $3.1 \AA$ to the donor. In the absence of the $\beta$-phosphate as in the case of FMP, the $\alpha$-phosphate is the only possibility for hydrogen bonding, and it is very likely that it will form the hydrogen bond with $Y 300 \beta$ considering that the phosphate can have at most 2 negative charges. It is difficult to conclude that $Y 300 \beta$ facilitates the farnesylation by specifically hydrogen bonding with the $\alpha$-phosphate of the substrate simply because a similar drop in $k_{\text {chem }}$ was observed. We speculate that the transition state may have a partially departed phosphate leaving group and that $Y 300 \beta$ helps to stabilize the developing negative charge through hydrogen bonding. This hypothesis is further supported by the potential of mean force calculations discussed below. 


\section{The Potential of Mean Force Calculations}

The various free energy profiles of the conformational activation step were computed as a function of the distance between the center of mass of atoms C1, C2, and O1 of $\mathrm{FPP}^{3-}$ or $\mathrm{FPP}^{2-}$ and $\mathrm{S}_{\gamma}$ of $\mathrm{Cys}^{2}$ of the target peptides and are shown in Figure 3. The statistical uncertainties of the calculated profiles are in general less than $0.2 \mathrm{kcal} / \mathrm{mol}$ except in the region where the distance is near $4.5 \AA$ and the sampling becomes more difficult because of potential van der Waals clashes. The influence of having one extra negative charge on the diphosphate upon the energetic cost of the transition is apparent when the free energy profiles of FTase/ $\mathrm{FPP}^{2-} / \mathrm{CVIM}$ and FTase/FPP ${ }^{3-} / \mathrm{CVIM}$ are compared. Breaking the salt bridge interactions

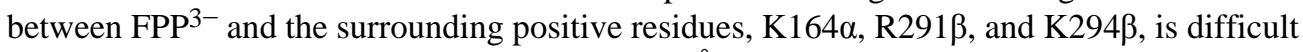
with a free energy penalty of nearly $5.0 \mathrm{kcal} /(\mathrm{mol} / \mathrm{A})$. No additional minima were found along the entire range of the reaction coordinate other than the one at $7.5 \AA$ that corresponds to the inactive conformation. With the monoprotonated farnesyldiphosphate, however, closing the gap between the two reaction centers by displacing the diphosphate from its binding pocket becomes energetically feasible. Separated by a small free energy barrier (ca. $1.0 \mathrm{kcal} / \mathrm{mol}$ ), one stable intermediate state was located at approximately $5.0 \AA, 0.4 \mathrm{kcal} / \mathrm{mol}$ higher than the FPP resting state. At the intermediate state, the hydrogen bonding and salt bridging patterns of the diphosphate are perturbed from those of the resting state marked by the formation of a few hydrogen bonds, including $\mathrm{H} 248 \beta$ with the $\alpha$-phosphate, $\mathrm{K} 164 \alpha$ with the capping acetyl group of CVIM, and K294 $\beta$ with the $\beta$-phosphate. The conformation of the farnesyl group at the intermediate state is remarkably similar to that found in the farnesylated product (Figure 4), the crystal structure of which is available (PDB ID: 1KZP) (19), particularly the alignment of the first and the second isoprene units and the orientation of their methyl groups. At the resting state, the methyl group of the first isoprene unit, to which the diphosphate is attached, is docked in an aromatic pocket consisting of Y166 $\alpha, \mathrm{Y} 200 \alpha, \mathrm{H} 201 \alpha, \mathrm{H} 248 \beta$, and Y $251 \beta$, but is "unlocked" upon closing the gap. At the intermediate state, the first and the second isoprene units rest against $\mathrm{Val}^{2}$ and $\mathrm{Ile}^{3}$ of the peptide respectively with their methyl groups pointing in the same direction. Farthest from the gap, the terminal isoprene unit is deeply buried in the hydrophobic pocket formed by W102 $\beta$, Y154 $\beta$, Y205 $\beta, \mathrm{C} 254 \beta$, W $303 \beta$ and $\mathrm{Val}^{2}$, the interactions with which appear to be unaltered. With the diphosphate hydrogen bonding and salt bridge interactions largely intact, we speculate that the small free energy cost computed for the conformational transition is a result of dislodging the first isoprene unit from its binding pocket and the relocation of solvent molecules in the active site. The identification of this neartarget intermediate state and the associated low free energy profile directly supports the isoprene-rotation mechanism previously proposed based on crystal structure information only.

The recently published kinetic experiments conducted by Fierke and coworkers (23) measured the reaction rate constants of radio-active FPP and two different peptide targets, TKCVIF and GCVLS. The observed differential kinetic isotope effects imply peptide "target-dependent" energetics of the conformational activation step, an interesting and unprecedented discovery, which can be directly probed computationally. Instead of attempting an exact comparison, which would require two fresh sets of relatively expensive umbrella MD simulations for TKCVIF and GCVIS, we only tested the effect of mutation at the third position of the CaaX motif based on our analyses on the structural details along the transition path, which suggest that residues outside of the Caa box are unlikely to have significant impact on the free energy profile. From the crystal structure of FTase complexed with TKCVIF and an FPP analog (PDB ID: 1TN7) (38), the threonine and lysine are located beyond the farnesyl binding site and exposed to the solvent. The $\mathrm{X}$ position of the CaaX motif is buried in the same binding site, but the side chain does not form close contact with the farnesyl group.

We computed the free energy profile for CVLM, which was readily done by adapting the snapshots for CVIM and mutating the isoleucine (the most critical position to the activation in 
the CaaX motif) to leucine while the methionine was preserved. This resulted in a relatively small perturbation to the local structure, which can be quickly accommodated. The result (dashed line in Figure 3) features the same resting state and the intermediate state. However, the intermediate state is about $1.0 \mathrm{kcal} / \mathrm{mol}$ higher in free energy compared to that of CVIM and is separated from the resting state by an energy barrier that is $1.5 \mathrm{kcal} / \mathrm{mol}$ higher, which suggests a 4.5 -fold decrease in the conformational transition rate constant. Although the elevated transition barrier as a result of a conservative mutation in the third position of the CaaX motif qualitatively supports the interpretation of the observed target-dependent $\alpha$ secondary ${ }^{3} \mathrm{H}$-KIEs, which was attributed to a higher barrier for the conformational activation step for GCVLS versus TKCVIF, this change may not be significant enough to cause a shift in the rate-determining step from the chemical step (for TKCVIF) to the conformational activation step (foe GCVLS). Certainly, the other non-Caa residues of the peptides may also affect the transition, but their contributions are likely even smaller because they do not appear to directly interact with the farnesyl group and their conformations are not noticeably altered. We hypothesize that other factors are responsible for the apparent differences in the observed KIEs for the two cases, which will be further investigated using QM/MM reaction path and computational KIE studies.

The average structures of all the umbrella MD trajectories were computed for both peptides and are available in the supplementary material. The conformations of the farnesyl group and all the residues that are within $5 \AA ̊$ of FPP were similar throughout the reaction coordinate. The filled substrate binding pocket limits the possibility for dramatic differences in the orientation of critical residues. The small difference in the barrier heights for CVIM and CVLM is most likely the consequence of short-range interactions, which slightly favors the choice of an

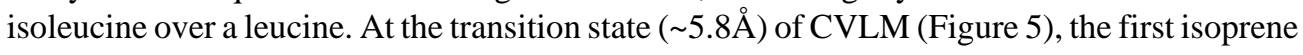
unit of FPP has already been dislocated from the hydrophobic pocket and assumes the same orientation as found in the intermediate state, but the transition of the second isoprene unit has not been completed. Most interestingly, the farnesyl group deviates from the fully extended conformation at the transition state with a kink developing between the second and the third isoprene units. The average binding site conformation of CVIM at the same reaction coordinate point is almost identical; however, the kinked farnesyl group, particularly the third isoprene unit, interacts better with the side chain of $\mathrm{Ile}^{3}$, which explains the reduced conformational free energy.

In the PMF calculations carried out for CVIM and CVLM with the wild-type FTase, we observed that Y300 $\beta$ acted as the donor of the hydrogen bond with the $\beta$-diphosphate of FPP throughout the entire reaction profile. We hypothesized that the catalytic power of Y300 $\beta$ ( $\sim 500$-fold decrease in $k_{\text {chem }}$ upon mutation) is not associated with the FPP activation step. To investigate, we computed the corresponding free energy profile for the conformational activation of the Y300F $\beta$ mutant FTase and CVIM (the dotted line in Figure 3). Eliminating the possibility of hydrogen bonding does not significantly affect the free energy of the conformational transition, but shifts both the intermediate state and the resting state farther away from the zinc-bound cysteine of the peptide, indicating that the measured reduction in the reaction rate upon this mutation is likely from the chemical reaction step in which Y $300 \beta$ may participate in the stabilization of the leaving diphosphate group.

\section{CONCLUSIONS}

The molecular-level details of the mechanism of FTase catalyzed prenylation have been obfuscated due to the apparent necessity of a significant conformational rearrangement of the substrate prior to the chemical step. This makes it difficult to interpret even the most carefully thought out kinetic experiments, because the measured rate constants reflect the kinetics of at least two processes that may or may not have differential activation free energies depending 
on the peptide substrate. In this second part of our computational effort aimed at unraveling some of the remaining mechanistic issues in FTase, we focused on exploring the thermodynamics of the conformational activation step, removed from complications associated with the subsequent chemical reaction steps.

Combining our results with available experimental findings, we are able to present a plausible scenario of the key events prior to the chemical reaction in FTase. At physiological $\mathrm{pH}$, both $\mathrm{FPP}^{3-}$ and $\mathrm{FPP}^{2-}$ may exist in solution as well as in complex with FTase. However, the $\mathrm{FPP}^{2-}$ form of the ternary complex is most likely the only productive form in the absence of magnesium, as we found closing the $7 \AA$ gap energetically prohibitive for fully deprotonated FPP owing to the highly (positively) charged diphosphate binding pocket. One major intermediate state was identified as a result of the PMF calculations, in which the two reaction sites are approximately 5.0 $\mathrm{A}$ from each other. The conformation of the farnesyl group of the intermediate, particularly the first two isoprene units, are in excellent agreement with that found in the product complex. During the transition, the diphosphate group is able to maintain the salt bridge interactions with the positively charged side chains from K164 $\alpha$, R291 $\beta$ and $\mathrm{K} 294 \beta$ as well as the hydrogen bonds with $\mathrm{H} 248 \beta$ and $\mathrm{Y} 300 \beta$. In addition, most of the binding site residues, including the ones from the peptide targets, are minimally perturbed by the conformational change to the farnesyl group, which explains the small energy cost associated with the transition.

The identification and characterization of the intermediate state at a relatively close distance supports the farnesyl-attacking mechanism, rather than the thiolate-attacking mechanism, but also allowed us to examine the effects of mutations on the thermodynamics of the conformational activation step. We found that changing the peptide target from CVIM to CVLM increased the transition free energy barrier height by about $1.5 \mathrm{kcal} / \mathrm{mol}$. Although qualitatively consistent with the findings from recent KIE experiments of Fierke and coworkers, the magnitude is not significant enough to warrant a shift of the rate-determining step from the chemistry step to the conformational transition step, which was used to interpret the observed KIE measurements for two different peptide substrates. The details of the subsequent chemical steps are now under investigation utilizing $\mathrm{QM} / \mathrm{MM}$ techniques.

\section{Supplementary Material}

Refer to Web version on PubMed Central for supplementary material.

\section{ACKNOWLEDGMENT}

The authors thank NIH for financial support of this project through a grant (GM44974) to K.M.M.

\section{REFERENCES}

1. Adjei AA. Blocking oncogenic Ras signaling for cancer therapy. J. Natl. Cancer I 2001;93:1062-1074.

2. Resh M. Regulation of cellular signaling by fatty acid acylation and prenylation of signal transduction proteins. Cell. Signaling 1996;8:403-412.

3. Roskoski R. Protein prenylation: a pivotal posttranslational process. Biochem. Bioph. Res. Co 2003;303:1-7.

4. Seabra M. Membrane association and targeting of prenylated Ras-like GTPases. Cell. Signaling 1998;10:167-172.

5. Sinensky M. Recent advances in the study of prenylated proteins. Bba-Mol. Cell. Biol. L 2000;1484:93-106.

6. Zhang FL, Casey PJ. Annu. Rev. Biochem 1996;65:241-269. [PubMed: 8811180] 
7. Caponigro F, Casale M, Bryce J. Farnesyl transferase inhibitors in clinical development. Expert Opin. Inv. Drug 2003;12:943-954.

8. Doll RJ, Kirschmeier P, Bishop WR. Farnesyltransferase inhibitors as anticancer agents: Critical crossroads. Curr. Opin. Drug. Disc 2004;7:478-486.

9. Huang CY, Rokosz L. Farnesyltransferase inhibitors: recent advances. Expert Opin. Ther. Pat 2004;14:175-186.

10. Khuri FR, Glisson BS, Kim ES, Statkevich P, Thall PF, Meyers ML, et al. Phase I study of the farnesyltransferase inhibitor lonafarnib with paclitaxel in solid tumors. Clin. Cancer Res 2004;10:2968-2976. [PubMed: 15131032]

11. Rao S, Cunningham D, de Gramont A, Scheithauer W, Smakal M, Humblet Y, et al. Phase III doubleblind placebo-controlled study of farnesyl transferase inhibitor R115777 in patients with refractory advanced colorectal cancer. J. Clin. Oncol 2004;22:3950-3957. [PubMed: 15459217]

12. Taveras AG, Kirschmeier P, Baum CM. SCH-66336 (Sarasar (R)) and other benzocycloheptapyridyl farnesyl protein transferase inhibitors: Discovery, biology and clinical observations. Curr. Top. Med. Chem 2003;3:1103-1114. [PubMed: 12769711]

13. Hightower KE, De S, Weinbaum C, Spence RA, Casey PJ. Lysine(164)alpha of protein farnesyltransferase is important for both CaaX substrate binding and catalysis. Biochem. J 2001;360:625-631. [PubMed: 11736652]

14. Wu Z, Demma M, Strickland CL, Radisky ES, Poulter CD, Le HV, Windsor WT. Farnesyl protein transferase: Identification of K164 alpha and Y300 beta as catalytic residues by mutagenesis and kinetic studies. Biochem 1999;38:11239-11249. [PubMed: 10471273]

15. Pickett JS, Bowers KE, Hartman HL, Fu HW, Embry AC, Casey PJ, Fierke CA. Kinetic studies of protein farnesyltransferase mutants establish active substrate conformation. Biochem 2003;42:97419748. [PubMed: 12911316]

16. Dunten P, Kammlott U, Crowther R, Weber D, Palermo R, Birktoft J. Protein farnesyltransferase: Structure and implications for substrate binding. Biochem 1998;37:7907-7912. [PubMed: 9609683]

17. Long SB, Casey PJ, Beese LS. Cocrystal structure of protein farnesyltransferase complexed with a farnesyl diphosphate substrate. Biochem 1998;37:9612-9618. [PubMed: 9657673]

18. Long SB, Casey PJ, Beese LS. The basis for K-Ras4B binding specificity to protein farnesyltransferase revealed by 2 angstrom resolution ternary complex structures. Struct. Fold. Des 2000;8:209-222.

19. Long SB, Casey PJ, Beese LS. Reaction path of protein farnesyltransferase at atomic resolution. Nature 2002;419:645-650. [PubMed: 12374986]

20. Long SB, Hancock PJ, Kral AM, Hellinga HW, Beese LS. The crystal structure of human protein farnesyltransferase reveals the basis for inhibition by CaaX tetrapeptides and their mimetics. Proc. Natl. Acad. Sci. USA 2001;98:12948-12953. [PubMed: 11687658]

21. Park HW, Boduluri SR, Moomaw JF, Casey PJ, Beese LS. Crystal structure of protein farnesyltransferase at 2.25 angstrom resolution. Science 1997;275:1800-1804. [PubMed: 9065406]

22. Strickland CL, Windsor WT, Syto R, Wang L, Bond R, Wu Z, Schwartz J, Le HV, Beese LS, Weber PC. Crystal structure of farnesyl protein transferase complexed with a CaaX peptide and farnesyl diphosphate analogue. Biochem 1998;37:16601-16611. [PubMed: 9843427]

23. Pais JE, Bowers KE, Fierke CA. Measurement of the alpha-secondary kinetic isotope effect for the reaction catalyzed by mammalian protein farnesyltransferase. J. Am. Chem. Soc 2006;128:1508615087. [PubMed: 17117849]

24. Lenevich SXJ, Hosokawa A, Cramer CJ, Distefano MD. Transition state analysis of model and enzymatic prenylation reactions. J. Am. Chem. Soc 2007;129:5796-5797. [PubMed: 17439118]

25. Daan Frenkel, BS. Understanding Molecular Simulation: From Algorithms to Applications. 2 ed.. Vol. 1. Academic Press; 2002.

26. Cui G, Wang B, Merz KM Jr. Computational studies of the farnesyltransferase ternary complex part I: substrate binding. Biochem 2005;44:16513-16523. [PubMed: 16342942]

27. Saderholm MJ, Hightower KE, Fierke CA. Role of metals in the reaction catalyzed by protein farnesyltransferase. Biochem 2000;39:12398-12405. [PubMed: 11015220]

28. Case DA, Darden TA, Cheatham TE III, Simmerling CL, Wang J, Duke RE, et al. AMBER 8. 2004 
29. Frisch MJ, Trucks GW, Schlegel HB, Scuseria GE, Robb MA, Cheeseman JR. Gaussian 03. 2003

30. Bayly CI, Cieplak P, Cornell WD, Kollman PA. A well-behaved electrostatic potential based method using charge restraints for deriving atomic charges - the RESP model. J. Phys. Chem 1993;97:1026910280.

31. Wang JM, Wolf RM, Caldwell JW, Kollman PA, Case DA. Development and testing of a general amber force field. J. Comput. Chem 2004;25:1157-1174. [PubMed: 15116359]

32. Kumar S, Bouzida D, Swendsen R, Kollman P, Rosenberg J. The weighted histogram analysis method for free-energy calculations on biomolecules .1. the method. J. Comput. Chem 1992;13:1011-1021.

33. Cornell WD, Cieplak P, Bayly CI, Gould IR, Merz KM, Ferguson DM, Spellmeyer DC, Fox T, Caldwell JW, Kollman PA. A 2nd generation force-field for the simulation of proteins, nucleic-acids, and organic-molecules. J. Am. Chem. Soc 1995;117:5179-5197.

34. Berendsen HJC, Postma JPM, Vangunsteren WF, Dinola A, Haak JR. Molecular-dynamics with coupling to an external bath. J. Chem. Phys 1984;81:3684-3690.

35. Andrea TA, Swope WC, Andersen HC. The role of long ranged forces in determining the structure and properties of liquid water. J. Chem. Phys 1983;79:4576-4584.

36. Grossfield, A. Weighted histogram analysis method. 2003. http://dasher.wustl.edu/alan/wham/

37. Roux B. The calculation of the potential of mean force using computer simulations. Comput. Phys. Comm 1995;91:275-282.

38. Reid TS, Terry KL, Casey PJ, Beese LS. Crystallographic analysis of CaaX prenyltransferases complexed with substrates defines rules of protein substrate selectivity. J. Mol. Biol 2004;343:417433. [PubMed: 15451670] 


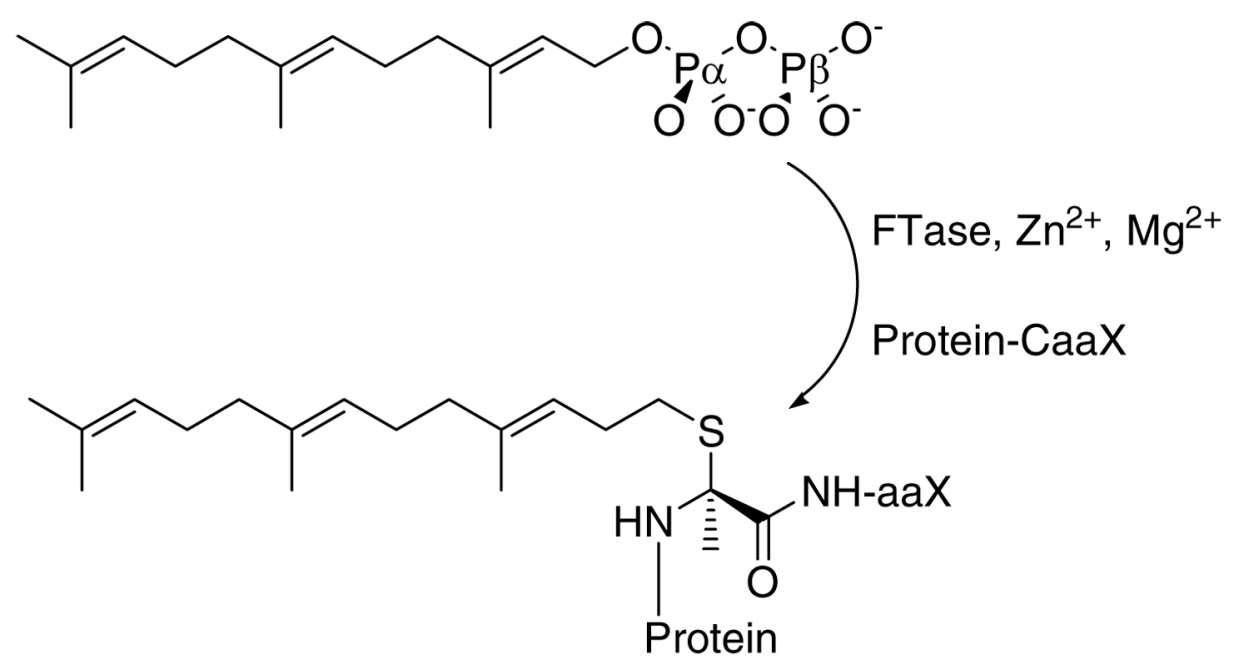

Figure 1.

The reaction catalyzed by farnesyltransferase. 


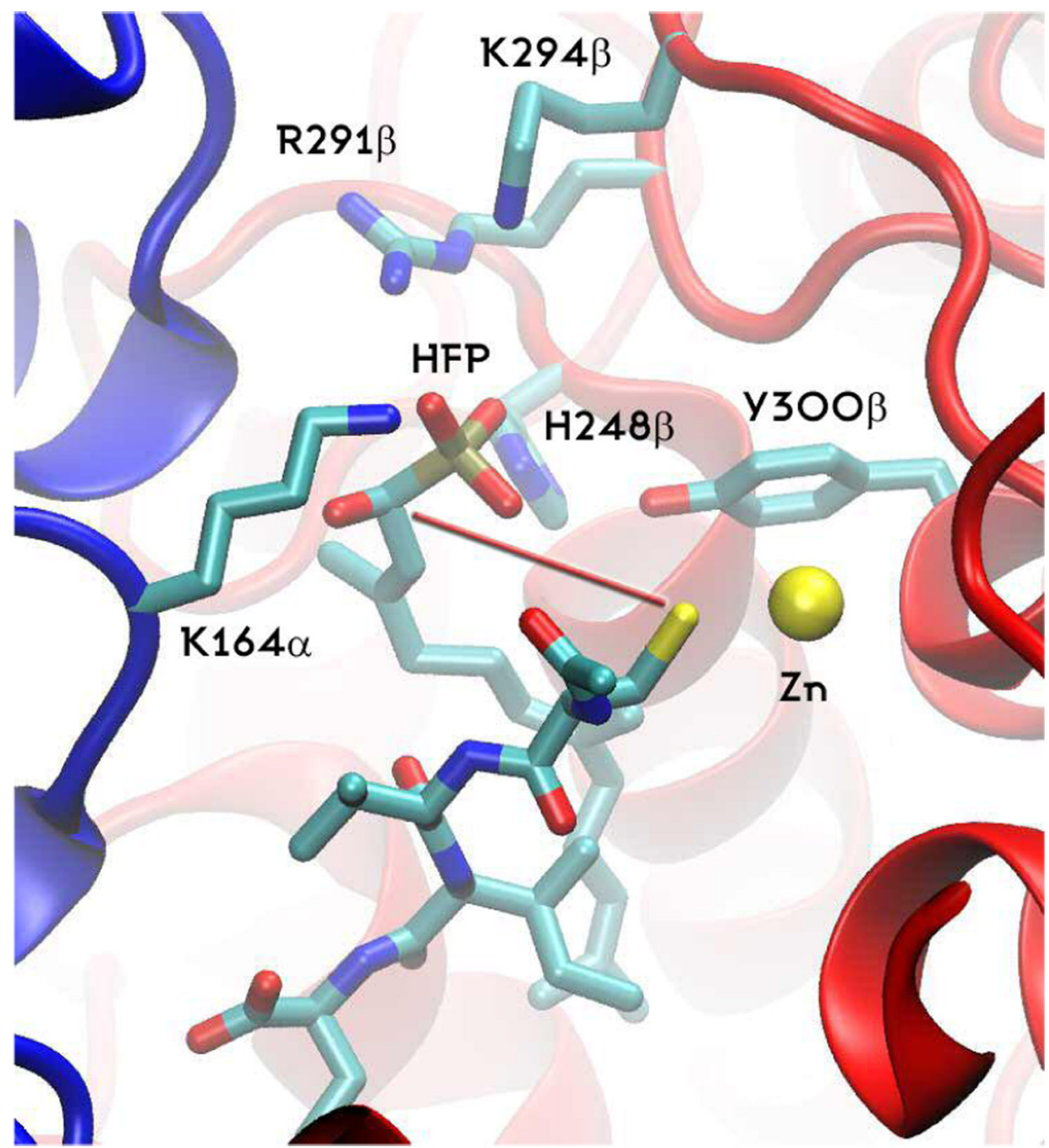

Figure 2.

The substrate binding site as seen in the crystal structure 1QBQ. Zinc, HFP, CVIM and HFP binding residues are shown as licorice. $\alpha$ (blue) and $\beta$ (red) monomers are represented as ribbon diagram. The $7.2 \AA$ gap between the attacking nucleophile and the farnesyl acceptor is highlighted with a red line. 


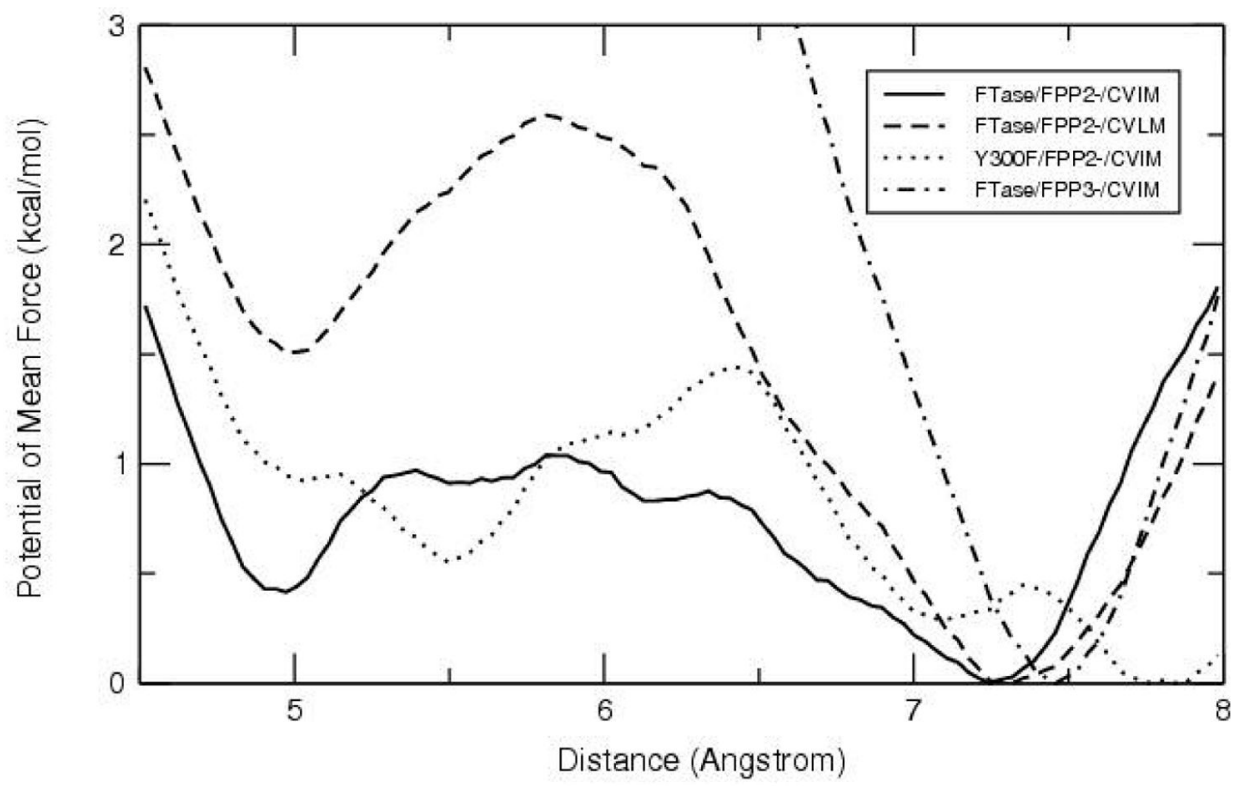

Figure 3.

The free energy profiles of the conformational activation step in FTase ternary complexes computed as a function of the distance between the center of mass of atoms $\mathrm{C} 1, \mathrm{C} 2$, and $\mathrm{O} 1$ of $\mathrm{FPP}^{3-}$ or $\mathrm{FPP}^{2-}$ and $\mathrm{S}_{\gamma}$ of $\mathrm{Cys}^{2}$ of the target peptides. The full PMF curve of FTase/ $\mathrm{FPP}^{3-} / \mathrm{CVIM}$ is truncated at $3.0 \mathrm{kcal} / \mathrm{mol}$ to fit into plot of the other PMFs. 


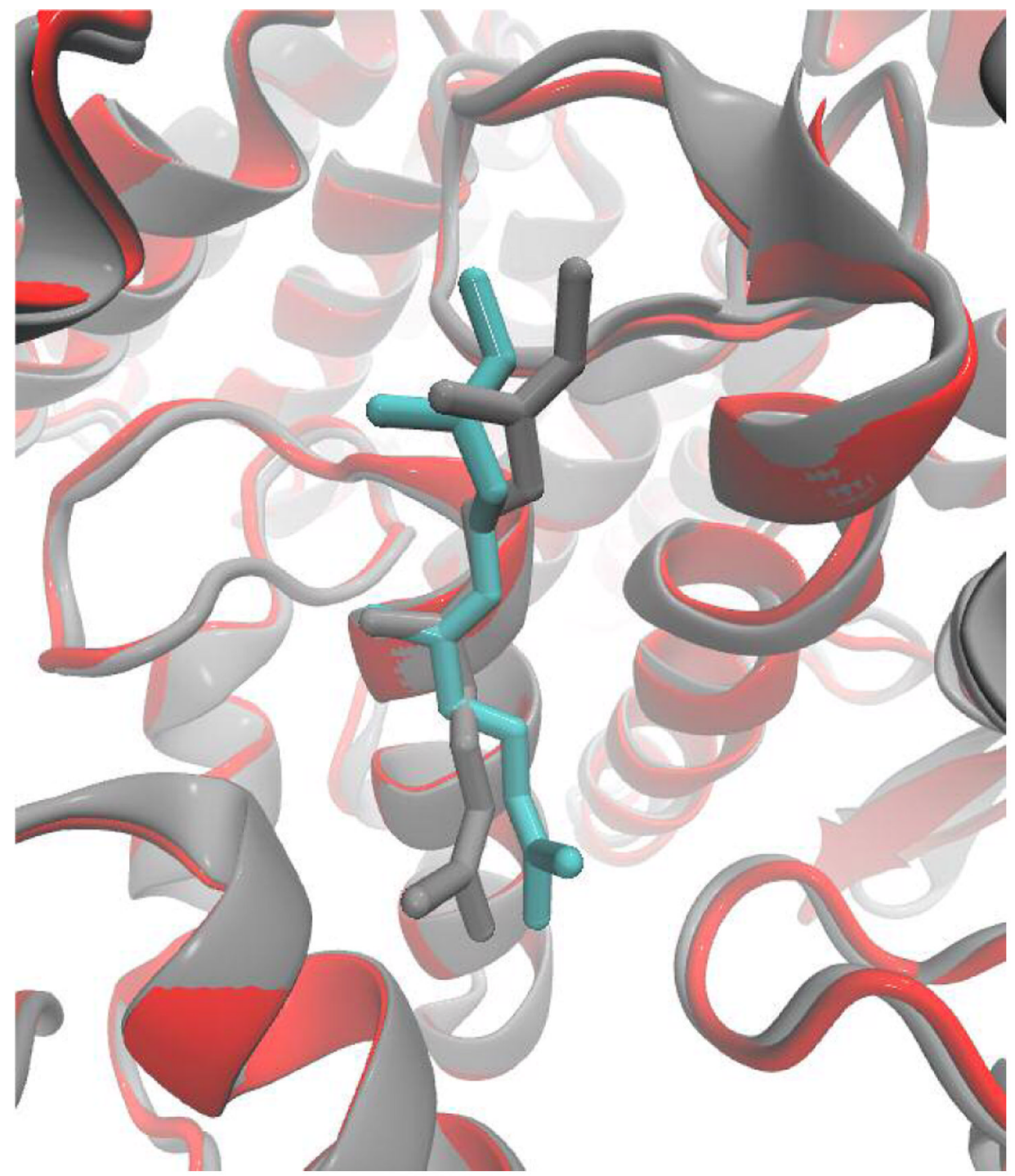

Figure 4.

The average conformation of the farnesyl group at the intermediate state (cyan) and that found in 1KZP (grey) are shown in licorice with the backbone conformations (ribbons) of FTase superimposed. The monoprotonated diphosphate and the farnesylated peptide are omitted for clarity. 


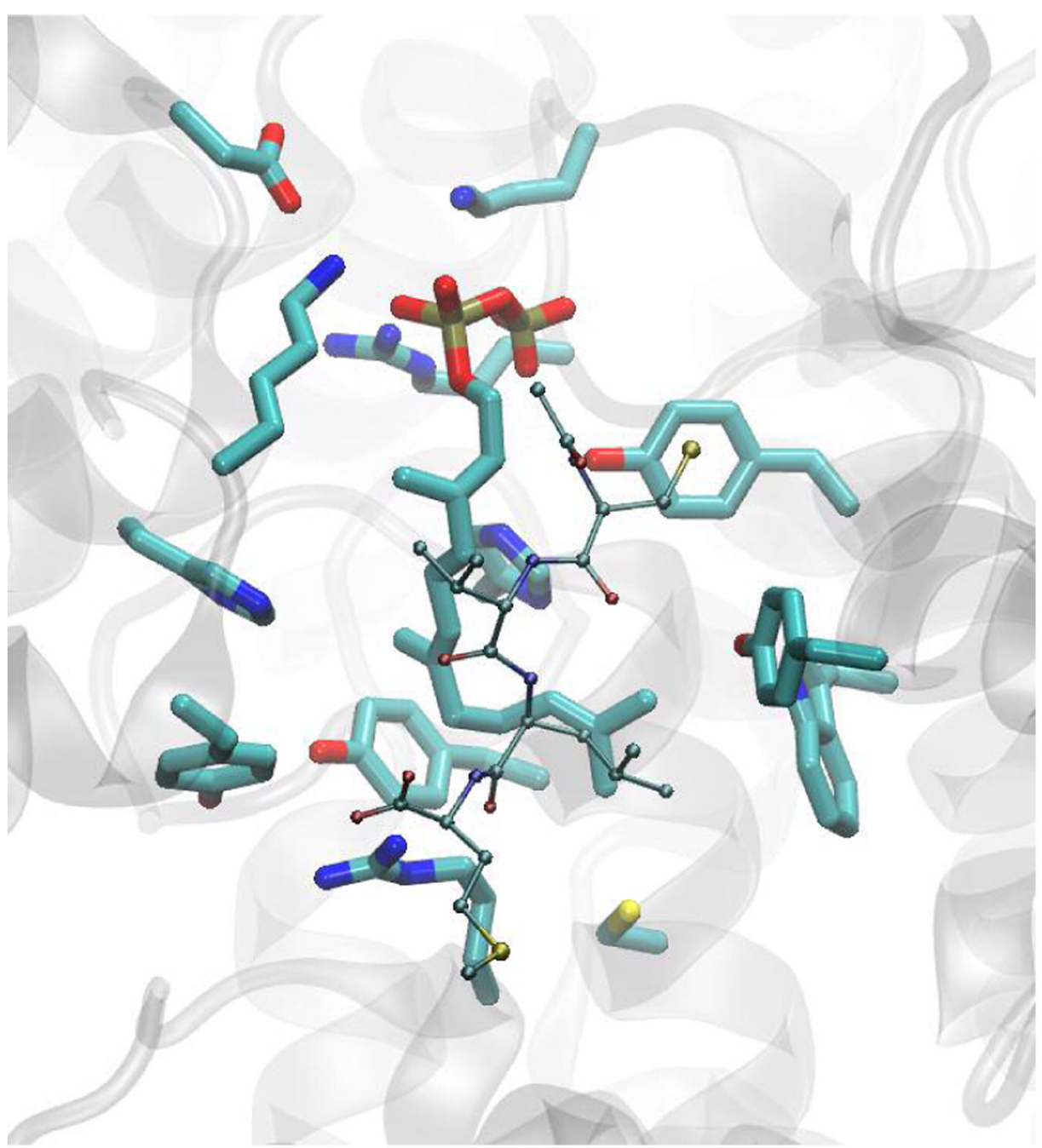

Figure 5.

The average transition state conformation of CVLM (shown in CPK), FPP, and close residues (shown in Licorice). 